\title{
Study on Genetic Parameters and Performance of Garden Pea (Pisum sativum L.) Genotypes for Yield and its Components (Under Northern Transitional Belt of Karnataka, India)
}

\author{
Imran Ali $^{1}{ }^{*}$, R.V. Patil ${ }^{1}$, P.R. Dharmatti ${ }^{1}$ and O. Sridevi ${ }^{2}$ \\ ${ }^{1}$ Department of Horticulture, ${ }^{2}$ Department of Genetics and Plant Breeding, College of \\ Agriculture Dharwad, University of Agricultural Sciences (UAS), \\ Dharwad, Karnataka - 580 005, India \\ *Corresponding author
}

\section{Keywords}

Garden pea, Genetic advance, Heritability, Variability, GCV and PCV

\section{Article Info}

Accepted: 08 August 2018 Available Online: 10 September 2018

\section{A B S T R A C T}

The present investigation was carried out to study genetic parameters and performance of thirty four and forty eight genotypes of garden pea in kharif and rabi season, respectively, during 2017. Genotypes were grown in RBD design with two replications at Main Agricultural Research Station, UAS, Dharwad. Analysis of variance revealed significant differences among the genotypes, for all the characters studied, indicating the presence of considerable amount of variability in the material. During kharif season, the genotypes viz., DWDP-0007, DWDP-0023, Arkel, AP-3 and IIPR 45-UB-40 recorded high mean performance for pod yield per plant, pod length and number of seeds per pod. During rabi season, the genotypes viz., Jhunjhunu Matar, DWDP-0003, IIPR 45-UB30, Kashi Agethi and VRP-5 recorded high mean performance for pod yield per plant, pod length, number of seeds per pod and weight of 100 fresh seeds. Hence, these genotypes could be utilized in the hybridization programmes to bring pod yield improvement in garden pea. The estimates of genetic parameters revealed that GCV and PCV were high for days to first flowering, plant height, days to $50 \%$ flowering (during both seasons), pod yield (during kharif), weight of hundred fresh and dry seeds (during rabi), indicating presence of high variability and less influence of environment on their expression. High heritability (broad sense) coupled with high genetic advance as per cent of mean was observed for plant height, days to first flowering, days to $50 \%$ flowering, number of branches per plant (during both seasons), pod yield (during kharif), pod length, weight of hundred fresh and dry seeds (during rabi), indicating that simple selection would be helpful for the improvement of these characters as these are governed by additive gene action. Number of pods per plant, number of seeds per pod (during kharif), number of seeds per pod and protein content (during rabi) showed moderate heritability and genetic advance.

\section{Introduction}

Garden pea (Pisum sativum L.) $(2 \mathrm{n}=2 \mathrm{x}=14)$ is one of the most important leguminous vegetable grown for their delicious, nutritious seeds throughout the world. It belongs to the family Fabaceae and sub family papilionaceae. In India, pea is cultivated on an area of 4.98 
lakh ha with a production of 48.11 lakh tonne and the productivity is $9.70 \mathrm{t} / \mathrm{ha}$. India ranks $1^{\text {st }}$ in the production of pea. Uttar Pradesh stands first in area and production. It is 2.18 lakh ha area and production is 24.81 lakh tonne, followed by Madhya Pradesh and Jharkhand. In Karnataka, area and production is about 1.54 thousand ha and 20.37 thousand tonne respectively, with a productivity of 13.26 t/ha (Anonymous, 2016).

Pea is known because of its rich nutritive value. It is a rich source of carbohydrate, protein, vitamins (A and C) and minerals like calcium and magnesium. According to National Institute of Nutrition, Hyderabad nutritive value of green pea (per $100 \mathrm{~g}$ of edible portion) is: protein- $7.20 \mathrm{~g}$, carbohydrate- $15.90 \mathrm{~g}$, fat- $0.10 \mathrm{~g}$, fiber- 4.00 $\mathrm{g}$, calcium- $20.00 \mathrm{mg}$, magnesium- $34.00 \mathrm{mg}$, carotene- $83.00 \mu \mathrm{g}$, thiamine- $0.25 \mathrm{mg}$, riboflavin- $0.01 \mathrm{mg}$, niacin- $0.80 \mathrm{mg}$.

High fiber content in pea improves bowel health and peristalsis. The niacin in peas helps to reduce the production of triglycerides, which results in less bad cholesterol. The dried and powdered seed has been used as a poultice on the skin, where it has an appreciable effect on many types of skin complaint including acne and wrinkled skin. Different compounds present in pea like coumestrol, pisum saponins I and II, pisimosides A and B and phenolic acids helps in prevention of stomach cancer.

The major constraints of pea production include low productivity due to nonavailability of location specific varieties suitable for year round cultivation. Hence, there is an urgent need to evaluate the germplasm of pea to select high yielding genotypes which can be adopted as such for commercial production or can be incorporated in the future breeding programmes for improvement of yield. Estimates of parameters of variability importantly, heritability and genetic gain are reliable indicators for improvement of characters in a particular genetic material through selection. Keeping these in view, the present study was taken up to estimate the genetic parameters in gaeden pea genotypes to identify the superior genotypes for pod yield.

\section{Materials and Methods}

The research was conducted at Network Project on Onion and Garlic site, Department of Horticulture, Main Agricultural Research Station, University of Agricultural Sciences, Dharwad, in kharif and rabi season, during 2017. The experimental material for the present investigation consisted of thirty four and fourty eight garden pea genotypes, during kharif and rabi season, respectively. The experiment was laid in RBD design with two replications with spacing of $45 \mathrm{~cm}$ between rows and $30 \mathrm{~cm}$ between plants. Five plants were selected at random from each replication and data were recorded for characters viz., plant height, days to first flowering, days to 50 per cent flowering, number of branches per plant, number of pods per plant, pod yield per plant, pod length, pod width and number of seeds per pod for both seasons. Observation on weight of hundred fresh and dry seeds, protein content and total soluble solids content was recorded during rabi season only. Genetic parameters like variance, genotypic and phenotypic coefficient of variation, heritability and genetic advance as per cent of mean were calculated as per the standard procedure given by Singh and Choudhary (1979).

\section{Results and Discussion}

Analysis of variance revealed significant differences among the thirty four and fourty eight genotypes, for all the ten and fourteen characters studied during kharif and rabi season respectively, indicating the presence of considerable amount of variability in the 
material (Table 1 and 2). The genotypes DWDP-0007, DWDP-0023, Arkel, AP-3 and IIPR 45-UB-40 recorded high mean performance for pod yield per plant, pod length and number of seeds per pod during kharif season (Table 3). During rabi season (Table 4), number of pods per plant was found maximum in genotype DWDP-0009, pod length was reorded maximum in genotype Kashi Agethi, number of seeds per pod and weight of hundred fresh seeds was found highest in genotype VRP-5. Pod yield per plant was found highest in genotypes Jhunjhunu Matar, DWDP-0003, IIPR-45-UB30, Kashi Agethi and Kedia Matar.

Based on the mean performance of genotypes, genotypes DWDP-0007, AP-3, Arkel and IIPR-44-UB-40 were good performer during kharif season. The genotypes, Jhunjhunu Matar, Kashi Agethi and VRP-5 had good performance during rabi season. Hence, these genotypes can be selected as promising parent to develop high yielding progeny for yield improvement in garden pea.

The mean performance of all genotypes during kharif and rabi season, were studied. Investigation indicates significant differences for all the characters among the genotypes. Yield during both growing season was not good and low yield was found for all genotypes, because weather parameters (high temperature and low relative humidity) were not favorable for potential yield of the crop. During rabi season yield was comparatively high then kharif season, because of availability of comparatively cool and favorable climatic condition to boost the yield of garden pea.

In the present study, the estimates of PCV for all the characters were higher than the estimates of GCV, which may be due to the interaction of genotypes with the environment. During kharif season (Table 5), the highest estimates of coefficients of variation were registered for pod yield per plant followed by days to first flowering, plant height 40 DAS and days to fifty per cent flowering (Devi et $a l ., 2017)$. A higher estimate of genotypic and phenotypic coefficients of variation indicates the presence of ample variability among the genotypes for these characters. Similar finding were reported by Mehta et al., (2005), Lal et al., (2011) and Afreen et al., (2017), in garden pea.

The moderate estimates of GCV and PCV were recorded for number of pods per plant followed by plant height 75 DAS and pod length during kharif season, indicating the less variability among the genotypes for these characters. These results are in agreement with the findings of Sureja et al., (2000), Singh et al., (2003), Ramesh et al., (2002) and Chaudhary et al., (2010), in garden pea.

During rabi season (Table 6), weight of hundred fresh seeds followed by plant height 60 DAS, weight of hundred dry seeds and days to first flowering showed maximum phenotypic and genotypic coefficient of variation. A higher estimate of genotypic and phenotypic coefficients of variation indicates the presence of ample variability among the genotypes for these characters.

Therefore, simple selection for these characters could be effective for bringing further improvement in garden pea. These results are in accordance with the studies of Mehta et al., (2005), Lal et al., (2011) and Afreen et al., (2017), in garden pea.

Moderate estimates of GCV and PCV was observed for number of branches per plant followed by days to fifty per cent flowering, number of seeds per pod, pod length and TSS content during rabi season indicated the less variability among the genotypes for these characters. 
Table.1 Analysis of variance for ten characters in thirty four garden pea genotypes grown during kharif season

\begin{tabular}{|c|l|c|c|c|}
\hline Sl. No. & \multicolumn{1}{|c|}{ Character } & \multicolumn{3}{|c|}{ Mean sum of squares } \\
\cline { 2 - 4 } & & $\begin{array}{c}\text { Replication } \\
(\mathbf{d f :} \mathbf{1})\end{array}$ & $\begin{array}{c}\text { Genotype } \\
\text { (df: 33) }\end{array}$ & $\begin{array}{c}\text { Error } \\
\text { (df: 33) }\end{array}$ \\
\hline 1 & Plant height 40 DAS (cm) & 33.82 & $178.93 * *$ & 4.29 \\
\hline 2 & Plant height 75 DAS (cm) & 52.95 & $142.47 * *$ & 11.27 \\
\hline 3 & Days to first flowering & 15.05 & $288.63 * *$ & 18.27 \\
\hline 4 & Days to 50 \% flowering & 7.77 & $285.76^{* *}$ & 17.84 \\
\hline 5 & No. of branches per plant & 2.11 & $1.43 * *$ & 0.20 \\
\hline 6 & No. of pods per plant & 2.60 & $17.31 * *$ & 6.43 \\
\hline 7 & Pod length (cm) & 0.04 & $1.29 * *$ & 0.20 \\
\hline 8 & Pod width (cm) & 0.01 & $0.01 * *$ & 0.00 \\
\hline 9 & No. of seeds per pod & 0.14 & $1.23 * *$ & 0.37 \\
\hline 10 & Pod yield per plant $(\mathrm{g})$ & 31.79 & $74.09 * *$ & 15.39 \\
\hline
\end{tabular}

* Significant at 5\% level; ** Significant at $1 \%$ level

Table.2 Analysis of variance for fourteen characters in fourty eight garden pea genotypes grown during rabi season

\begin{tabular}{|c|c|c|c|c|}
\hline \multirow{3}{*}{$\begin{array}{l}\text { Sl. } \\
\text { No. }\end{array}$} & \multirow[t]{3}{*}{ Character } & \multicolumn{3}{|c|}{ Mean sum of squares } \\
\hline & & Replication & Genotype & Error \\
\hline & & (df: 1$)$ & (df: 47) & (df:47) \\
\hline 1 & Plant height 60 DAS $(\mathrm{cm})$ & 0.91 & $366.01 * *$ & 36.83 \\
\hline 2 & Plant height 90 DAS (cm) & 178.76 & $344.97 * *$ & 38.98 \\
\hline 3 & Days to first flowering & 5.04 & $235.95 * *$ & 7.63 \\
\hline 4 & Days to $50 \%$ flowering & 0.66 & $221.58 * *$ & 6.94 \\
\hline 5 & No. of branches per plant & 7.04 & $0.90 * *$ & 0.19 \\
\hline 6 & No. of pods per plant & 45.54 & $11.57 * *$ & 4.21 \\
\hline 7 & Pod length $(\mathrm{cm})$ & 0.10 & $1.98 * *$ & 0.14 \\
\hline 8 & Pod width $(\mathrm{cm})$ & 0.06 & $0.02 * *$ & 0.00 \\
\hline 9 & No. of seeds per pod & 0.16 & $1.58 * *$ & 0.43 \\
\hline 10 & Weight of 100 fresh seeds $(\mathrm{g})$ & 4.90 & $153.49 * *$ & 0.17 \\
\hline 11 & Weight of 100 dry seeds (g) & 3.97 & $17.17 * *$ & 0.10 \\
\hline 12 & TSS content $\left({ }^{\circ}\right.$ brix $)$ & 1.40 & $23.99 * *$ & 0.30 \\
\hline 13 & Protein content $(\%)$ & 0.02 & $4.85 * *$ & 1.33 \\
\hline 14 & Pod yield per plant (g) & 49.95 & $82.68 * *$ & 39.90 \\
\hline
\end{tabular}

* Significant at 5\% level; ** Significant at $1 \%$ level 
Table.3 Mean performance of thirty four genotypes of garden pea (Pisum sativum L.) for ten characters grown during kharif season

\begin{tabular}{|c|c|c|c|c|c|c|c|c|c|c|c|}
\hline $\begin{array}{l}\text { SI. } \\
\text { No. }\end{array}$ & Genotypes & $\begin{array}{c}\text { Plant } \\
\text { height } \\
40 \text { DAS } \\
(\mathrm{cm})\end{array}$ & $\begin{array}{c}\text { Plant } \\
\text { height } \\
75 \text { DAS } \\
(\mathrm{cm})\end{array}$ & $\begin{array}{c}\text { Days to } \\
\text { first } \\
\text { flowering }\end{array}$ & $\begin{array}{c}\text { Days to } \\
50 \% \\
\text { flowerin } \\
\mathrm{g}\end{array}$ & $\begin{array}{c}\text { No. of } \\
\text { branche } \\
\text { s per } \\
\text { plant }\end{array}$ & $\begin{array}{l}\text { No. of } \\
\text { pods } \\
\text { per } \\
\text { plant }\end{array}$ & $\begin{array}{c}\text { Pod } \\
\text { length } \\
(\mathrm{cm})\end{array}$ & $\begin{array}{l}\text { Pod width } \\
(\mathrm{cm})\end{array}$ & $\begin{array}{c}\text { No. of } \\
\text { seeds } \\
\text { per } \\
\text { pod }\end{array}$ & $\begin{array}{c}\text { Pod } \\
\text { yield } \\
\text { per } \\
\text { plant (g) }\end{array}$ \\
\hline 1 & DWDP-0001 & 15.50 & 43.84 & 48.00 & 53.00 & 5.00 & 17.17 & 7.32 & 1.11 & 6.95 & 16.50 \\
\hline 2 & DWDP-0002 & 19.17 & 48.83 & 39.50 & 43.50 & 3.50 & 17.84 & 7.14 & 1.11 & 6.95 & 19.67 \\
\hline 3 & DWDP-0003 & 15.50 & 35.50 & 39.00 & 44.00 & 4.00 & 17.84 & 7.19 & 1.01 & 6.67 & 27.67 \\
\hline 4 & DWDP-0004 & 33.67 & 64.00 & 24.00 & 33.50 & 6.00 & 23.67 & 4.93 & 0.84 & 5.23 & 18.50 \\
\hline 5 & DWDP-0005 & 30.67 & 63.67 & 46.50 & 51.00 & 5.00 & 21.84 & 6.46 & 1.12 & 5.67 & 18.83 \\
\hline 6 & DWDP-0006 & 38.34 & 60.50 & 49.00 & 55.00 & 4.00 & 20.83 & 6.23 & 1.10 & 6.45 & 21.84 \\
\hline 7 & DWDP-0007 & 47.50 & 58.00 & 43.50 & 49.50 & 4.50 & 23.84 & 6.10 & 1.02 & 5.84 & 30.34 \\
\hline 8 & DWDP-0008 & 45.83 & 64.00 & 58.00 & 65.50 & 5.00 & 23.67 & 6.18 & 1.07 & 6.56 & 14.67 \\
\hline 9 & DWDP-0009 & 38.67 & 58.00 & 25.00 & 33.00 & 4.50 & 25.83 & 5.70 & 0.92 & 5.89 & 12.17 \\
\hline 10 & DWDP-0011 & 39.33 & 57.84 & 59.00 & 65.00 & 5.50 & 21.50 & 6.48 & 1.18 & 6.95 & 26.33 \\
\hline 11 & DWDP-0012 & 38.83 & 58.84 & 53.50 & 60.00 & 5.00 & 20.67 & 6.37 & 1.07 & 6.95 & 21.34 \\
\hline 12 & DWDP-0014 & 46.34 & 70.34 & 59.00 & 70.50 & 6.00 & 22.50 & 5.98 & 0.99 & 6.12 & 18.34 \\
\hline 13 & DWDP-0015 & 46.84 & 64.00 & 47.50 & 54.00 & 5.50 & 20.17 & 6.12 & 1.04 & 6.17 & 22.09 \\
\hline 14 & DWDP-0018 & 33.17 & 63.17 & 49.00 & 55.50 & 5.50 & 20.17 & 6.46 & 1.19 & 5.73 & 12.17 \\
\hline 15 & DWDP-0019 & 54.67 & 71.17 & 52.00 & 59.50 & 4.50 & 25.00 & 5.83 & 1.14 & 5.61 & 11.84 \\
\hline 16 & DWDP-0021 & 40.50 & 58.33 & 58.50 & 63.50 & 5.00 & 23.83 & 6.01 & 1.15 & 6.22 & 21.00 \\
\hline 17 & DWDP-0023 & 25.67 & 51.50 & 36.00 & 41.00 & 5.50 & 18.17 & 8.10 & 1.03 & 8.11 & 30.34 \\
\hline 18 & GS-10 & 33.17 & 56.83 & 35.50 & 41.00 & 5.50 & 20.17 & 7.42 & 1.07 & 7.22 & 27.50 \\
\hline 19 & PSM-13 & 21.84 & 47.67 & 24.50 & 30.50 & 4.50 & 14.67 & 7.49 & 1.22 & 7.56 & 25.67 \\
\hline 20 & Kedia Matar & 37.17 & 54.00 & 32.50 & 40.00 & 6.00 & 17.00 & 7.35 & 1.03 & 7.72 & 26.67 \\
\hline 21 & Kashi Agethi & 29.17 & 49.17 & 24.00 & 31.50 & 5.00 & 15.17 & 8.05 & 1.16 & 8.23 & 27.50 \\
\hline 22 & Arkel & 29.33 & 44.34 & 23.00 & 28.50 & 4.50 & 15.00 & 8.13 & 1.20 & 8.06 & 29.34 \\
\hline 23 & AP-1 & 33.17 & 50.50 & 33.50 & 40.00 & 4.00 & 20.17 & 7.63 & 1.22 & 7.78 & 19.00 \\
\hline 24 & AP-3 & 19.00 & 44.67 & 24.00 & 32.00 & 3.50 & 15.50 & 7.52 & 1.24 & 7.34 & 29.50 \\
\hline 25 & VRP-5 & 24.50 & 41.84 & 23.00 & 30.50 & 4.50 & 15.50 & 7.89 & 1.18 & 7.72 & 24.50 \\
\hline 26 & VRP-6 & 29.50 & 48.00 & 23.50 & 32.00 & 3.00 & 17.50 & 7.45 & 1.21 & 6.78 & 17.17 \\
\hline 27 & PC-531 & 34.67 & 48.50 & 35.50 & 41.50 & 4.50 & 19.17 & 7.10 & 1.03 & 6.39 & 20.84 \\
\hline
\end{tabular}


Table.3 (Conti...)

\begin{tabular}{|c|c|c|c|c|c|c|c|c|c|c|c|}
\hline $\begin{array}{l}\text { Sl. } \\
\text { No. }\end{array}$ & Genotypes & $\begin{array}{c}\text { Plant } \\
\text { height } \\
40 \\
\text { DAS } \\
\text { (cm) }\end{array}$ & $\begin{array}{c}\text { Plant } \\
\text { height } \\
75 \\
\text { DAS } \\
\text { (cm) }\end{array}$ & $\begin{array}{l}\text { Days to } \\
\text { first } \\
\text { floweri } \\
\text { ng }\end{array}$ & $\begin{array}{c}\text { Days to } \\
50 \% \\
\text { flowerin } \\
\mathrm{g}\end{array}$ & $\begin{array}{l}\text { No. of } \\
\text { branch } \\
\text { es per } \\
\text { plant }\end{array}$ & $\begin{array}{c}\text { No. of } \\
\text { pods } \\
\text { per } \\
\text { plant }\end{array}$ & $\begin{array}{c}\text { Pod } \\
\text { length } \\
(\mathrm{cm})\end{array}$ & $\begin{array}{l}\text { Pod } \\
\text { width } \\
\text { (cm) }\end{array}$ & $\begin{array}{c}\text { No. of } \\
\text { seeds } \\
\text { per } \\
\text { pod }\end{array}$ & $\begin{array}{c}\text { Pod } \\
\text { yield } \\
\text { per } \\
\text { plant } \\
(\mathrm{g})\end{array}$ \\
\hline 28 & IIPR-37 & 28.17 & 46.17 & 56.50 & 63.50 & 4.00 & 19.83 & 6.65 & 1.16 & 6.61 & 16.34 \\
\hline 29 & IIPR-36 & 29.33 & 45.34 & 45.00 & 51.00 & 4.50 & 19.67 & 6.85 & 1.04 & 6.50 & 9.17 \\
\hline 30 & IIPR-43 & 29.00 & 49.67 & 38.00 & 42.50 & 3.00 & 18.83 & 7.25 & 1.10 & 6.34 & 18.34 \\
\hline 31 & IIPR- 44- UB-40 & 34.67 & 52.50 & 38.00 & 44.00 & 4.50 & 20.17 & 8.20 & 1.07 & 7.61 & 28.34 \\
\hline 32 & IIPR -45-UB-30 & 30.67 & 45.84 & 37.50 & 42.50 & 3.00 & 19.50 & 7.59 & 1.10 & 7.56 & 24.67 \\
\hline 33 & DWDP-0024 & 20.17 & 53.83 & 43.50 & 50.00 & 3.50 & 18.00 & 6.80 & 1.17 & 6.78 & 11.17 \\
\hline \multirow[t]{9}{*}{34} & 46- Local & 29.00 & 51.67 & 52.50 & 58.50 & 4.50 & 20.34 & 7.42 & 1.14 & 6.72 & 24.50 \\
\hline & Mean & 32.43 & 53.59 & 40.50 & 46.96 & 4.59 & 19.73 & 6.92 & 1.10 & 6.79 & 21.29 \\
\hline & $\begin{array}{c}\text { Range (Lowest to } \\
\text { Highest) }\end{array}$ & $\begin{array}{c}15.50 \\
\text { to } \\
54.67\end{array}$ & $\begin{array}{c}35.50 \\
\text { to } \\
71.17\end{array}$ & $\begin{array}{c}23.00 \text { to } \\
59.00\end{array}$ & $\begin{array}{c}28.50 \text { to } \\
70.50\end{array}$ & $\begin{array}{c}3.00 \text { to } \\
6.00\end{array}$ & $\begin{array}{c}14.67 \\
\text { to } \\
25.83\end{array}$ & $\begin{array}{c}4.93 \text { to } \\
8.20\end{array}$ & $\begin{array}{c}0.84 \text { to } \\
1.24\end{array}$ & $\begin{array}{l}5.23 \text { to } \\
8.23\end{array}$ & $\begin{array}{l}9.17 \text { to } \\
30.34\end{array}$ \\
\hline & C.V. (\%) & 6.39 & 6.27 & 10.55 & 9.00 & 9.95 & 12.86 & 6.54 & 2.48 & 9.05 & 18.43 \\
\hline & $\mathrm{F}$ ratio & 41.68 & 12.63 & 15.80 & 16.02 & 6.90 & 2.69 & 6.32 & 21.01 & 3.26 & 4.81 \\
\hline & F Prob. & 0.00 & 0.00 & 0.00 & 0.00 & 0.00 & 0.00 & 0.00 & 0.00 & 0.00 & 0.00 \\
\hline & S.Em. \pm & 1.47 & 2.37 & 3.02 & 2.99 & 0.32 & 1.79 & 0.32 & 0.02 & 0.43 & 2.77 \\
\hline & C.D. @ 5\% & 4.22 & 6.83 & 8.70 & 8.59 & 0.93 & 5.16 & 0.92 & 0.06 & 1.25 & 7.98 \\
\hline & C.D. @ 1\% & 5.66 & 9.18 & 11.68 & 11.54 & 1.25 & 6.94 & 1.24 & 0.07 & 1.68 & 10.73 \\
\hline
\end{tabular}


Table.4 Mean performance of fourty eight genotypes of garden pea (Pisum sativum L.) for fourteen characters grown during rabi season

\begin{tabular}{|c|c|c|c|c|c|c|c|c|c|c|c|c|c|c|c|}
\hline $\begin{array}{l}\text { Sl. } \\
\text { No. }\end{array}$ & Genotypes & $\begin{array}{c}\text { Plant } \\
\text { height } \\
60 \text { DAS } \\
(\mathrm{cm})\end{array}$ & $\begin{array}{c}\text { Plant } \\
\text { height } \\
90 \text { DAS } \\
(\mathrm{cm})\end{array}$ & $\begin{array}{l}\text { Days to } \\
\text { first } \\
\text { flowe- } \\
\text { ring }\end{array}$ & $\begin{array}{l}\text { Days to } \\
50 \% \\
\text { flowe- } \\
\text { ring }\end{array}$ & $\begin{array}{c}\text { No. of } \\
\text { branc- } \\
\text { hes per } \\
\text { plant }\end{array}$ & $\begin{array}{c}\text { No. of } \\
\text { pods per } \\
\text { plant }\end{array}$ & $\begin{array}{c}\text { Pod } \\
\text { length } \\
(\mathrm{cm})\end{array}$ & $\begin{array}{l}\text { Pod } \\
\text { width } \\
(\mathrm{cm})\end{array}$ & $\begin{array}{c}\text { No. of } \\
\text { seeds } \\
\text { per } \\
\text { pod }\end{array}$ & $\begin{array}{l}\text { Weight } \\
\text { of } 100 \\
\text { fresh } \\
\text { seeds }(\mathrm{g})\end{array}$ & $\begin{array}{c}\text { Weight } \\
\text { of } 100 \\
\text { dry } \\
\text { seeds (g) }\end{array}$ & $\begin{array}{c}\text { TSS } \\
\text { content } \\
\left({ }^{\circ} \text { brix) }\right.\end{array}$ & $\begin{array}{l}\text { Protein } \\
\text { content } \\
(\%)\end{array}$ & $\begin{array}{c}\text { Pod } \\
\text { yield } \\
\text { per } \\
\text { plant } \\
\text { (g) }\end{array}$ \\
\hline 1 & DWDP-0001 & 41.83 & 59.67 & 49.50 & 54.00 & 5.00 & 9.33 & 6.68 & 1.08 & 5.83 & 38.70 & 18.00 & 27.10 & 13.82 & 19.17 \\
\hline 2 & DWDP-0002 & 36.50 & 58.34 & 47.00 & 51.50 & 4.50 & 10.72 & 7.42 & 1.03 & 5.56 & 30.20 & 10.60 & 32.25 & 10.79 & 24.84 \\
\hline 3 & DWDP-0003 & 32.34 & 54.33 & 47.00 & 52.00 & 4.00 & 10.22 & 7.44 & 1.01 & 5.89 & 29.75 & 9.15 & 23.90 & 14.11 & 35.95 \\
\hline 4 & DWDP-0004 & 52.34 & 68.50 & 32.00 & 40.50 & 5.50 & 18.39 & 4.74 & 0.75 & 5.45 & 16.10 & 8.65 & 31.60 & 13.91 & 24.89 \\
\hline 5 & DWDP-0005 & 69.67 & 85.33 & 62.50 & 67.50 & 5.00 & 11.84 & 5.63 & 1.11 & 5.34 & 20.75 & 9.40 & 24.15 & 16.62 & 20.89 \\
\hline 6 & DWDP-0006 & 60.00 & 75.00 & 62.50 & 68.00 & 4.50 & 13.28 & 5.74 & 1.06 & 5.95 & 23.00 & 10.55 & 33.60 & 15.23 & 26.06 \\
\hline 7 & DWDP-0007 & 60.67 & 83.33 & 63.00 & 68.00 & 4.00 & 12.67 & 5.33 & 0.95 & 5.11 & 24.05 & 8.60 & 25.00 & 13.19 & 28.61 \\
\hline 8 & DWDP-0008 & 67.33 & 85.17 & 52.50 & 59.50 & 6.00 & 16.11 & 5.39 & 0.98 & 5.67 & 14.25 & 13.90 & 26.95 & 14.11 & 27.33 \\
\hline 9 & DWDP-0009 & 53.84 & 78.00 & 31.50 & 38.50 & 3.50 & 21.39 & 4.33 & 0.77 & 5.33 & 14.75 & 7.40 & 23.55 & 16.36 & 23.89 \\
\hline 10 & DWDP-0010 & 36.34 & 55.17 & 63.00 & 69.50 & 3.50 & 10.39 & 6.51 & 1.17 & 5.28 & 35.05 & 13.15 & 26.35 & 15.29 & 17.67 \\
\hline 11 & DWDP-0011 & 67.17 & 90.50 & 61.00 & 66.50 & 4.50 & 13.95 & 5.92 & 1.12 & 6.39 & 25.30 & 11.60 & 25.15 & 16.24 & 32.39 \\
\hline 12 & DWDP-0012 & 73.83 & 91.50 & 57.50 & 64.50 & 5.00 & 15.84 & 5.61 & 1.05 & 5.39 & 16.10 & 13.90 & 31.10 & 14.00 & 27.45 \\
\hline 13 & DWDP-0013 & 51.50 & 61.50 & 51.00 & 57.00 & 3.00 & 13.84 & 5.70 & 1.09 & 5.39 & 23.05 & 13.25 & 24.35 & 15.21 & 26.00 \\
\hline 14 & DWDP-0014 & 65.50 & 85.34 & 62.50 & 68.50 & 4.00 & 12.62 & 5.67 & 0.99 & 5.61 & 20.55 & 8.05 & 27.60 & 12.77 & 20.56 \\
\hline 15 & DWDP-0015 & 68.83 & 89.50 & 62.00 & 68.00 & 4.00 & 13.72 & 5.67 & 0.98 & 5.34 & 22.05 & 17.10 & 26.90 & 12.78 & 27.84 \\
\hline 16 & DWDP-0016 & 56.17 & 76.67 & 67.50 & 72.00 & 3.50 & 11.89 & 5.22 & 0.96 & 5.73 & 20.90 & 17.70 & 28.70 & 14.66 & 17.17 \\
\hline 17 & DWDP-0017 & 55.83 & 76.67 & 56.50 & 64.00 & 4.00 & 12.11 & 6.02 & 1.07 & 5.83 & 35.40 & 15.50 & 25.70 & 15.88 & 19.23 \\
\hline 18 & DWDP-0018 & 72.17 & 94.00 & 57.00 & 63.50 & 4.50 & 12.61 & 5.40 & 0.97 & 5.17 & 26.95 & 13.65 & 26.80 & 16.63 & 16.28 \\
\hline 19 & DWDP-0019 & 68.67 & 93.50 & 55.00 & 62.50 & 4.00 & 14.45 & 5.38 & 1.05 & 6.34 & 30.60 & 14.50 & 19.45 & 13.93 & 25.06 \\
\hline 20 & DWDP-0020 & 46.50 & 66.17 & 47.00 & 55.00 & 3.00 & 13.61 & 5.55 & 1.03 & 6.34 & 20.60 & 11.25 & 23.20 & 15.65 & 15.95 \\
\hline 21 & DWDP-0021 & 65.67 & 85.67 & 59.50 & 67.00 & 4.00 & 13.22 & 5.60 & 1.09 & 6.06 & 21.60 & 10.65 & 24.60 & 16.51 & 25.06 \\
\hline 22 & DWDP-0022 & 50.00 & 66.67 & 65.50 & 71.50 & 4.00 & 12.28 & 6.07 & 1.15 & 5.89 & 19.50 & 10.50 & 29.45 & 14.43 & 20.84 \\
\hline 23 & DWDP-0023 & 38.00 & 60.50 & 47.00 & 52.00 & 3.50 & 9.89 & 7.68 & 1.01 & 7.67 & 41.55 & 13.50 & 30.30 & 15.34 & 34.06 \\
\hline 24 & GS-10 & 38.34 & 62.33 & 48.00 & 54.00 & 3.50 & 9.72 & 7.28 & 1.05 & 7.34 & 26.65 & 9.60 & 25.70 & 17.14 & 32.17 \\
\hline 25 & PSM-13 & 37.50 & 56.17 & 31.00 & 37.00 & 3.50 & 10.83 & 7.52 & 1.23 & 7.17 & 38.55 & 18.95 & 31.20 & 16.14 & 31.72 \\
\hline 26 & Udaipuri Matar & 54.17 & 72.34 & 61.50 & 66.50 & 4.00 & 11.61 & 5.94 & 1.14 & 5.67 & 28.65 & 14.65 & 29.40 & 13.94 & 20.84 \\
\hline 27 & Jhunjhunu Matar & 30.50 & 54.33 & 55.00 & 59.50 & 4.00 & 11.17 & 6.94 & 1.03 & 5.50 & 26.25 & 11.50 & 26.20 & 13.45 & 37.06 \\
\hline 28 & Kedia Matar & 35.17 & 55.17 & 47.50 & 55.00 & 3.50 & 9.89 & 7.12 & 1.01 & 6.84 & 36.25 & 11.45 & 28.20 & 14.37 & 35.00 \\
\hline 29 & Kashi Agethi & 37.00 & 59.33 & 35.00 & 42.50 & 3.00 & 10.95 & 8.15 & 1.20 & 7.67 & 42.05 & 17.85 & 31.70 & 16.08 & 35.23 \\
\hline 30 & Arkel & 40.17 & 64.17 & 31.50 & 37.00 & 3.50 & 9.62 & 7.83 & 1.22 & 7.89 & 40.95 & 14.80 & 29.70 & 18.39 & 32.73 \\
\hline 31 & DWDP-0025 & 54.83 & 75.00 & 66.00 & 72.50 & 3.50 & 11.67 & 6.69 & 1.16 & 6.23 & 23.05 & 10.20 & 28.30 & 15.15 & 26.06 \\
\hline 32 & DWDP-0026 & 37.34 & 59.84 & 47.00 & 55.00 & 3.00 & 10.56 & 7.59 & 1.17 & 7.45 & 28.75 & 11.45 & 30.30 & 16.71 & 21.95 \\
\hline 33 & DWDP-0027 & 29.34 & 51.84 & 66.00 & 73.00 & 4.00 & 10.10 & 7.40 & 1.06 & 7.38 & 26.91 & 9.49 & 25.72 & 17.64 & 32.59 \\
\hline 34 & AP-1 & 40.17 & 59.50 & 54.00 & 60.50 & 4.00 & 11.67 & 7.58 & 1.20 & 7.34 & 22.00 & 12.60 & 29.35 & 15.25 & 23.23 \\
\hline 35 & AP-3 & 40.00 & 60.67 & 34.00 & 42.00 & 3.50 & 9.45 & 7.44 & 1.21 & 7.28 & 44.50 & 11.70 & 30.25 & 17.26 & 31.06 \\
\hline
\end{tabular}


Table.4 (Cont...)

\begin{tabular}{|c|c|c|c|c|c|c|c|c|c|c|c|c|c|c|c|}
\hline $\begin{array}{l}\text { Sl. } \\
\text { No. }\end{array}$ & Genotypes & $\begin{array}{c}\text { Plant } \\
\text { heigh } \\
\text { t } 60 \\
\text { DAS } \\
(\mathrm{cm})\end{array}$ & $\begin{array}{c}\text { Plant } \\
\text { heig } \\
\text { ht } 90 \\
\text { DAS } \\
(\mathrm{cm})\end{array}$ & $\begin{array}{c}\text { Days to } \\
\text { first } \\
\text { floweri } \\
\text { ng }\end{array}$ & $\begin{array}{c}\text { Days to } \\
\mathbf{5 0 \%} \\
\text { floweri } \\
\text { ng }\end{array}$ & $\begin{array}{c}\text { No. of } \\
\text { branch } \\
\text {-es per } \\
\text { plant }\end{array}$ & $\begin{array}{l}\text { No. } \\
\text { of } \\
\text { pods } \\
\text { per } \\
\text { plant }\end{array}$ & $\begin{array}{c}\text { Pod } \\
\text { lengt } \\
\text { h } \\
(\mathrm{cm})\end{array}$ & $\begin{array}{c}\text { Pod } \\
\text { width } \\
\text { (cm) }\end{array}$ & $\begin{array}{c}\text { No. } \\
\text { of } \\
\text { seeds } \\
\text { per } \\
\text { pod }\end{array}$ & $\begin{array}{c}\text { Weight } \\
\text { of } 100 \\
\text { fresh } \\
\text { seeds } \\
\text { (g) }\end{array}$ & $\begin{array}{c}\text { Weight } \\
\text { of } 100 \\
\text { dry } \\
\text { seeds } \\
\text { (g) }\end{array}$ & $\begin{array}{c}\text { TSS } \\
\text { content } \\
\left({ }^{\circ} \text { brix }\right)\end{array}$ & $\begin{array}{c}\text { Prote } \\
\text { in } \\
\text { conte } \\
\text { nt } \\
(\%)\end{array}$ & $\begin{array}{c}\text { Pod } \\
\text { yield } \\
\text { per } \\
\text { plant } \\
\text { (g) }\end{array}$ \\
\hline 36 & VRP-5 & 36.17 & 57.00 & 31.50 & 39.00 & 4.50 & 10.56 & 8.14 & 1.18 & 8.11 & 46.50 & 16.95 & 32.65 & 16.55 & 31.12 \\
\hline 37 & VRP-6 & 33.33 & 57.00 & 39.50 & 47.50 & 3.50 & 9.56 & 7.25 & 1.20 & 7.23 & 44.55 & 13.90 & 28.80 & 14.85 & 26.45 \\
\hline 38 & PC-531 & 33.67 & 56.67 & 56.00 & 62.00 & 3.50 & 10.23 & 7.20 & 1.00 & 6.67 & 24.15 & 9.40 & 26.50 & 14.69 & 19.67 \\
\hline 39 & VL Matar-47 & 36.50 & 59.50 & 66.00 & 72.00 & 3.50 & 9.27 & 7.64 & 1.19 & 7.56 & 44.46 & 11.72 & 30.83 & 16.57 & 32.74 \\
\hline 40 & Local Collection & 48.33 & 69.84 & 66.00 & 72.50 & 4.00 & 10.83 & 5.77 & 0.97 & 5.78 & 18.15 & 12.65 & 35.60 & 16.02 & 12.78 \\
\hline 41 & IIPR-37 & 37.67 & 55.50 & 60.00 & 67.00 & 4.00 & 12.17 & 6.17 & 1.09 & 6.06 & 20.90 & 9.40 & 21.30 & 15.10 & 20.84 \\
\hline 42 & IIPR-36 & 35.67 & 53.50 & 62.50 & 68.50 & 3.00 & 12.06 & 6.49 & 1.04 & 6.34 & 22.85 & 11.40 & 23.75 & 19.27 & 16.23 \\
\hline 43 & IIPR-43 & 34.84 & 56.83 & 59.50 & 64.00 & 3.50 & 9.50 & 7.14 & 1.08 & 5.89 & 34.05 & 8.10 & 26.25 & 15.31 & 18.56 \\
\hline 44 & IIPR- 44- UB-40 & 34.67 & 55.33 & 48.50 & 54.50 & 3.00 & 10.89 & 8.08 & 1.04 & 7.84 & 23.55 & 9.60 & 21.85 & 15.74 & 27.67 \\
\hline 45 & IIPR -45-UB-30 & 36.17 & 57.84 & 50.00 & 55.00 & 3.00 & 9.34 & 7.61 & 1.03 & 6.67 & 26.05 & 9.75 & 22.80 & 17.07 & 35.67 \\
\hline 46 & IIPR-42-Local & 29.17 & 49.84 & 52.50 & 60.00 & 4.00 & 12.34 & 7.46 & 1.01 & 7.89 & 28.85 & 10.60 & 27.00 & 16.07 & 23.89 \\
\hline 47 & DWDP-0024 & 35.50 & 56.50 & 60.00 & 66.50 & 3.00 & 10.00 & 6.49 & 1.12 & 6.67 & 25.55 & 9.50 & 22.90 & 14.16 & 15.00 \\
\hline \multirow[t]{9}{*}{48} & 46- Local & 36.83 & 55.50 & 49.00 & 56.00 & 4.00 & 12.11 & 7.34 & 1.10 & 6.89 & 18.95 & 10.75 & 28.00 & 14.72 & 25.11 \\
\hline & Mean & 46.53 & 66.93 & 52.85 & 59.17 & 3.88 & 11.88 & 6.56 & 1.06 & 6.37 & 27.79 & 12.05 & 27.33 & 15.31 & 25.47 \\
\hline & $\begin{array}{l}\text { Range (Lowest to } \\
\text { Highest) }\end{array}$ & $\begin{array}{c}29.17 \\
\text { to } \\
73.83\end{array}$ & $\begin{array}{c}49.84 \\
\text { to } \\
94.00\end{array}$ & $\begin{array}{l}31.00 \\
\text { to } \\
67.50\end{array}$ & $\begin{array}{c}37.00 \text { to } \\
73.00\end{array}$ & $\begin{array}{c}3.00 \text { to } \\
6.00\end{array}$ & $\begin{array}{c}9.27 \\
\text { to } \\
21.39\end{array}$ & $\begin{array}{l}4.33 \\
\text { to } \\
8.15\end{array}$ & $\begin{array}{c}0.75 \\
\text { to } \\
1.23\end{array}$ & $\begin{array}{l}5.11 \\
\text { to } \\
8.11\end{array}$ & $\begin{array}{l}14.25 \\
\text { to } \\
46.50\end{array}$ & $\begin{array}{l}7.40 \text { to } \\
18.95\end{array}$ & $\begin{array}{c}19.45 \text { to } \\
35.60\end{array}$ & $\begin{array}{c}10.79 \\
\text { to } \\
19.27\end{array}$ & $\begin{array}{c}12.78 \\
\text { to } \\
37.06\end{array}$ \\
\hline & C.V. (\%) & 13.04 & 9.33 & 5.23 & 4.45 & 11.27 & 17.28 & 5.81 & 5.59 & 10.34 & 1.48 & 2.74 & 2.02 & 7.54 & 24.80 \\
\hline & F ratio & 9.94 & 8.85 & 30.89 & 31.91 & 4.74 & 2.75 & 13.70 & 5.81 & 3.65 & 901.78 & 157.10 & 79.01 & 3.64 & 2.07 \\
\hline & F Prob. & 0.00 & 0.00 & 0.00 & 0.00 & 0.00 & 0.00 & 0.00 & 0.00 & 0.00 & 0.00 & 0.00 & 0.00 & 0.00 & 0.01 \\
\hline & S.Em. \pm & 4.29 & 4.41 & 1.95 & 1.86 & 0.31 & 1.45 & 0.27 & 0.04 & 0.47 & 0.29 & 0.23 & 0.39 & 0.82 & 4.47 \\
\hline & C.D. @ $\overline{5 \%}$ & 12.21 & 12.56 & 5.56 & 5.30 & 0.88 & 4.13 & 0.77 & 0.12 & 1.33 & 0.83 & 0.67 & 1.11 & 2.32 & 12.71 \\
\hline & C.D. @ 1\% & 16.29 & 16.76 & 7.42 & 7.07 & 1.17 & 5.51 & 1.02 & 0.16 & 1.77 & 1.11 & 0.89 & 1.48 & 3.10 & 16.96 \\
\hline
\end{tabular}


Table.5 Mean, coefficient of variation, heritability (broad sense), genetic advance as per cent of mean for ten characters in garden pea (Pisum sativum L.) grown during kharif season

\begin{tabular}{|c|c|c|c|c|c|c|c|c|c|c|c|}
\hline \multirow[b]{2}{*}{$\begin{array}{l}\text { Sl. } \\
\text { No. }\end{array}$} & \multirow[b]{2}{*}{ Characters } & \multirow[b]{2}{*}{ Mean } & \multicolumn{2}{|c|}{ Range } & \multicolumn{2}{|c|}{ Variance } & \multicolumn{2}{|c|}{ Coefficient of variation } & \multirow{2}{*}{$\begin{array}{c}\text { Herita } \\
\text {-bility } \\
\text { (Broad } \\
\text { sense) } \\
(\%)\end{array}$} & \multirow{2}{*}{$\begin{array}{c}\text { Genetic } \\
\text { advance } \\
\text { (GA) }\end{array}$} & \multirow{2}{*}{$\begin{array}{c}\text { Genetic } \\
\text { advance as } \\
\text { per cent of } \\
\text { mean }(\%)\end{array}$} \\
\hline & & & Min. & Max. & Genotypic & Phenotypic & $\begin{array}{c}\text { Genotypic } \\
(\%)\end{array}$ & $\begin{array}{c}\text { Phenotypic } \\
(\%)\end{array}$ & & & \\
\hline 1. & $\begin{array}{l}\text { Plant height } \\
40 \text { DAS } \\
(\mathrm{cm})\end{array}$ & 32.43 & 15.50 & 54.67 & 87.32 & 91.61 & 28.81 & 29.51 & 95.31 & 18.79 & 57.94 \\
\hline 2. & $\begin{array}{l}\text { Plant height } \\
75 \text { DAS } \\
(\mathrm{cm})\end{array}$ & 53.59 & 35.50 & 71.17 & 65.60 & 76.87 & 15.11 & 16.36 & 85.33 & 15.41 & 28.76 \\
\hline 3. & $\begin{array}{l}\text { Days to first } \\
\text { flowering }\end{array}$ & 40.50 & 23.00 & 59.00 & 135.18 & 153.45 & 28.70 & 30.58 & 88.09 & 22.48 & 55.50 \\
\hline 4. & $\begin{array}{l}\text { Days to } 50 \\
\% \text { flowering }\end{array}$ & 46.96 & 28.50 & 70.50 & 133.96 & 151.80 & 24.64 & 26.23 & 88.25 & 22.39 & 47.70 \\
\hline 5. & $\begin{array}{l}\text { No. of } \\
\text { branches per } \\
\text { plant }\end{array}$ & 4.59 & 3.00 & 6.00 & 0.61 & 0.82 & 17.09 & 19.77 & 74.68 & 1.39 & 30.42 \\
\hline 6. & $\begin{array}{l}\text { No. of pods } \\
\text { per plant }\end{array}$ & 19.73 & 14.67 & 25.83 & 5.43 & 11.87 & 11.82 & 17.47 & 45.79 & 3.25 & 16.47 \\
\hline 7. & $\begin{array}{l}\text { Pod length } \\
(\mathrm{cm})\end{array}$ & 6.92 & 4.93 & 8.20 & 0.54 & 0.75 & 10.67 & 12.51 & 72.69 & 1.29 & 18.74 \\
\hline 8. & $\begin{array}{l}\text { Pod width } \\
(\mathrm{cm})\end{array}$ & 1.10 & 0.84 & 1.24 & 0.00 & 0.00 & 7.84 & 8.23 & 90.91 & 0.16 & 15.41 \\
\hline 9. & $\begin{array}{l}\text { No. of seeds } \\
\text { per pod }\end{array}$ & 6.79 & 5.23 & 8.23 & 0.42 & 0.80 & 9.62 & 13.21 & 53.10 & 0.98 & 14.45 \\
\hline 10. & $\begin{array}{l}\text { Pod yield } \\
\text { per plant (g) }\end{array}$ & 21.29 & 9.17 & 30.34 & 29.34 & 44.74 & 25.44 & 31.42 & 65.59 & 9.03 & 42.45 \\
\hline
\end{tabular}


Table.6 Mean, coefficient of variation, heritability (broad sense), genetic advance as per cent of mean for fourteen characters in garden pea (Pisum sativum L.) grown during rabi season

\begin{tabular}{|c|c|c|c|c|c|c|c|c|c|c|c|}
\hline \multirow{2}{*}{$\begin{array}{l}\text { Sl. } \\
\text { No. }\end{array}$} & \multirow[t]{2}{*}{ Characters } & \multirow[t]{2}{*}{ Mean } & \multicolumn{2}{|c|}{ Range } & \multicolumn{2}{|c|}{ Variance } & \multicolumn{2}{|c|}{ Coefficient of variation } & \multirow{2}{*}{$\begin{array}{c}\text { Herita } \\
\text {-bility } \\
\text { (Broad } \\
\text { sense) } \\
(\%)\end{array}$} & \multirow{2}{*}{$\begin{array}{c}\text { Genetic } \\
\text { advance } \\
\text { (GA) }\end{array}$} & \multirow{2}{*}{$\begin{array}{c}\text { Genetic } \\
\text { advance } \\
\text { as per } \\
\text { cent of } \\
\text { mean }(\%)\end{array}$} \\
\hline & & & Min. & Max. & Genotypic & Phenotypic & $\begin{array}{c}\text { Genotypic } \\
(\%)\end{array}$ & $\begin{array}{c}\text { Phenotypic } \\
(\%)\end{array}$ & & & \\
\hline 1. & $\begin{array}{l}\text { Plant height } 60 \text { DAS } \\
(\mathrm{cm})\end{array}$ & 46.53 & 29.17 & 73.83 & 164.59 & 201.42 & 27.56 & 30.49 & 81.71 & 23.89 & 51.33 \\
\hline 2. & $\begin{array}{l}\text { Plant height } 90 \text { DAS } \\
(\mathrm{cm})\end{array}$ & 66.93 & 49.84 & 94.00 & 152.99 & 191.97 & 18.48 & 20.70 & 79.69 & 22.47 & 33.98 \\
\hline 3. & $\begin{array}{l}\text { Days to first } \\
\text { flowering }\end{array}$ & 52.85 & 31.00 & 67.50 & 114.15 & 121.79 & 20.21 & 20.88 & 93.73 & 21.30 & 40.31 \\
\hline 4. & $\begin{array}{l}\text { Days to } 50 \% \\
\text { flowering }\end{array}$ & 59.17 & 37.00 & 73.00 & 107.32 & 114.26 & 17.50 & 18.06 & 93.92 & 20.68 & 34.95 \\
\hline 5 & $\begin{array}{l}\text { No. of branches per } \\
\text { plant }\end{array}$ & 3.88 & 3.00 & 6.00 & 0.35 & 0.54 & 15.41 & 19.09 & 65.18 & 0.99 & 25.63 \\
\hline 6. & No. of pods per plant & 11.88 & 9.27 & 21.39 & 3.67 & 7.89 & 16.14 & 23.64 & 46.61 & 2.69 & 22.70 \\
\hline 7. & Pod length $(\mathrm{cm})$ & 6.56 & 4.33 & 8.15 & 0.92 & 1.06 & 14.63 & 15.74 & 86.39 & 1.83 & 28.01 \\
\hline 8 & Pod width $(\mathrm{cm})$ & 1.06 & 0.75 & 1.23 & 0.00 & 0.01 & 8.66 & 10.30 & 70.62 & 0.15 & 14.99 \\
\hline 9. & No. of seeds per pod & 6.37 & 5.11 & 8.11 & 0.57 & 1.00 & 11.90 & 15.76 & 56.99 & 1.17 & 18.51 \\
\hline 10. & $\begin{array}{l}\text { Weight of } 100 \text { fresh } \\
\text { seeds }(\mathrm{g})\end{array}$ & 27.79 & 14.25 & 46.50 & 76.66 & 76.83 & 31.50 & 31.54 & 99.78 & 18.01 & 64.83 \\
\hline 11. & $\begin{array}{l}\text { Weight of } 100 \text { dry } \\
\text { seeds }(\mathrm{g})\end{array}$ & 12.05 & 7.40 & 18.95 & 8.83 & 8.64 & 24.23 & 24.38 & 98.74 & 5.97 & 49.60 \\
\hline 12. & TSS content ( ${ }^{\circ}$ brix $)$ & 27.33 & 19.45 & 35.60 & 11.84 & 12.14 & 12.59 & 12.75 & 97.50 & 7.00 & 25.61 \\
\hline 13. & Protein content $(\%)$ & 15.31 & 10.79 & 19.27 & 1.75 & 3.09 & 8.66 & 11.48 & 56.88 & 2.06 & 13.45 \\
\hline 14. & $\begin{array}{l}\text { Pod yield per plant } \\
\text { (g) }\end{array}$ & 25.47 & 12.78 & 37.06 & 21.38 & 61.29 & 18.16 & 30.74 & 34.90 & 5.62 & 22.09 \\
\hline
\end{tabular}


Between both seasons, compare to kharif season, more variability was observed in rabi season, for characters like number of pods per plant, pod length, pod width and number of seeds per pod.

All characters, exhibited relatively low magnitude of differences between PCV and GCV during both seasons, indicating less environmental influence on these characters. Thus, the characters showing maximum phenotypic coefficient of variation would be considered, while making selection in spite of influence of environment to a certain extent. These findings of genotypic and phenotypic coefficients of variation are in consonance with the reports of Sureja et al., (2000), Singh et al., (2003), Ramesh et al., (2002) and Chaudhary et al., (2010), in garden pea.

Heritability measures the relative amount of heritable portion of variability. It is a good index of the transmission of characters from parents to offspring. The perusal of the Table 5 , revealed the estimates of heritability in broad sense for ten characters studied during kharif seaon, which range from 45.79 to 95.31 per cent. High heritability was recorded for plant height 40 DAS followed by pod width, days to fifty per cent flowering (Tiwari and Roopalavanya, 2012; Afreen et al., 2017), days to first flowering (Habtamu and Million, 2013), plant height 75 DAS (Singh et al., 1993), number of branches per plant, pod length and pod yield per plant (Gudadinni et al., 2017). These results are in correspondences with the findings of Lavanaya et al., (2010), Lal et al., (2011), Gupta et al., (2006), Sharma et al., (2007), Guleria et al., (2009), Singh et al., (2011) and Sharma and Sharma (2013).

In rabi season (Table 6), highest heritability was observed for weight of 100 fresh seeds followed by weight of 100 dry seeds (Pratap et al., 1992 and Singh et al., 1993), TSS content, days to fifty per cent flowering (Tiwari and Roopalavanya, 2012), days to first flowering (Habtamu and Million, 2013), pod length, plant height 60 DAS, plant height 90 DAS (Singh et al., 1993), pod width and number of branches per plant. The result of high heritability estimates for above characters were also reported by Lavanaya $e t$ al., (2010), Lal et al., (2011), Gupta et al., (2006), Sharma et al., (2007), Guleria et al., (2009), Singh et al., (2011) and Sharma and Sharma (2013). This higher heritability value indicates that tentatively there might be additive gene effect for these characters. It showed that the characters having high value of heritability were least affected by the environmental modification and signified that the phenotypes were the true representative of their genotypes and selection based on phenotypic performance would be reliable.

Heritability estimates along with genetic advance are more useful than heritability alone in predicting the effectiveness of selection. Further, the heritability estimates coupled with expected genetic advance as per cent of mean indicates the mode of gene action in choosing an appropriate breeding methodology. During kharif season, high heritability coupled with high genetic advance as per cent of mean recorded for plant height (Gudadinni et al., 2017), days to first flowering, days to 50 per cent flowering, number of branches per plant and pod yield per plant indicates, additive gene action is involved in the genetic control of these traits. Hence, simple selection may help in improving these traits. These findings were well documented by Gupta et al., (2006) and Sharma et al., (2007), in garden pea.

During rabi season, maximum genetic advance were recorded for plant height 60 DAS, plant height 90 DAS (Lal et al., 2011 and Singh, 1995), days to first flowering and days to fifty per cent flowering. Moderate 
value of genetic advance was observed for weight of 100 fresh seeds. Higher heritability values along with high genetic advance indicated more scope exists for improvement of these characters by selection methods. These findings were well documented by Gupta et al., (2006) and Sharma et al., (2007), in garden pea.

During kharif season, moderate heritability and genetic advance as per cent of mean were recoded for number of pods per plant and number of seeds per pod. These findings were in consonance with the studies of Guleria et al., (2009) and Singh et al., (2011), in garden pea. High heritability coupled with moderate genetic advance as per cent of mean were recorded for pod length and pod width suggesting that the trait is controlled by both additive and non-additive gene action.

During rabi season, maximum genetic advance as per cent of mean was registered for weight of 100 fresh seeds followed by plant height 60 DAS, weight of 100 dry seeds, days to first flowering, days to fifty per cent flowering, plant height 90 DAS, pod length, number of branches per plant, TSS content, number of pods per plant and pod yield per plant (Gudadinni et al., 2017). Moderate values of genetic advance as per cent of mean were observed for number of seeds per pod, pod width, and protein content. These results indicated additive gene effects controlling the inheritance of these characters and simple selection schemes would be sufficient for the improvement of such characters. These findings were in consonance with the studies of Guleria et al., (2009) and Singh et al., (2011), in garden pea.

From the above discussion it can be concluded that, high estimates of GCV, PCV, heritability (broad sense) and genetic advance as per cent of mean were recorded for plant height, days to first flowering, days to 50 per cent flowering, pod yield per plant during both seasons, kharif and rabi. These results are in line with the findings of Pallavi and Pandey (2013) for days to 50 per cent flowering and pod yield per plant, indicating that simple selection would be helpful for the improvement of these traits as these are governed by additive gene action. Characters like number of pods per plant (during kharif) and number of seeds per pod (during both seasons), recoded moderate heritability and modrate genetic advance as per cent of mean and suggesting that characters are governed by both additive and non-additive gene action.

\section{Acknowledgements}

The authors are thankful to University of Agricultural Sciences (UAS), Dharwad to providing facilities for successful completion of research and Dr. Basamma for arrangement of material to conduct experiment successfully.

\section{References}

Afreen, S., Singh, A. K., Moharana, D. P., Singh, V., Singh, P. and Singh, B., 2017, Genetic evaluation for yield and yield attributes in garden pea (Pisum sativum var. hortense L.) under North Indian gangetic plain conditions. Int. J. Curr. Microbiol. App. Sci., 6(2): 13991404.

Anonymous, 2016, Indian horticulture database, http:www.nhb.gov.in.

Chaudhary, H., Verma, M. K. and Sofi, A. A., 2010, Genetic variability, heritability and genetic advance for yield components in garden pea. Pantnagar J. Res., 8(2): 195-197.

Devi, S., Kumar, M., Thakur, K., Bharat, N. K., Dogra, R. K. and Nagar, A., 2017, Variability, correlation and path analysis in pea (Pisum sativum L.) genotypes under Western Himalayan 
conditions. Chem. Sci. Rev. Lett., 6(21), 555-560.

Gudadinni, P., Bahadur, V., Ligade, P., Topno, S. E. and Prasad, V. M., 2017, Study on genetic variability, heritability and genetic advance in garden pea (Pisum sativum var. hortense L.). Int. J. Curr. Microbiol. App. Sci., 6(8): 23842391.

Guleria, S., Chongtham, N. and Dua, S., 2009, Genetic variability, correlation and path analysis studies in pea (Pisum sativum L.). Crop Res., 38(2): 179-183.

Gupta, A. J., Singh, Y. V. and Verma, T. S., 2006, Genetic variability and heritability in garden pea (Pisum sativum L.). Indian J. Hort., 63(3): 332334.

Habtamu, S. and Million, F., 2013, Multivariate analysis of some Ethiopian field pea (Pisum sativum L.) genotypes. Int. J. Genet. Mol. Biol., 78-87.

Lal, G. M., Meena, M. L., Chandra, K. and Singh, C. M., 2011, Assessment of genetic variability and interrelation between yield and its contributing components in field pea (Pisum sativum L.). Envt. and Eco., 29(3A): 1235-1239.

Lavanya, G. R., Singh, D. and Vinoth, R., 2010, Genetic variability, character association and component analysis in fieldpea, (Pisum sativum var. arvense L.). Madras Agri. J., 97(10/12): 329331.

Mehta, S., Kohli, U. K., Devinder, M. and Kumar, D., 2005, Genetic variability studies in pea (Pisum sativum L.). Haryana J. Hort. Sci., 34(1/2): 140-141.

Pallavi, A. S. and Pandey, K. K., 2013, Estimation of heritability on pea (Pisum sativum L.). Ad. Biores., 4: 89-92.

Pratap, P. S., Bhatia, G. L. and Arora, S. K., 1992, Studies on genetic variability and heritability for yield and component characters in pea (Pisum sativum L.). Crop Res., 5(3): 505-511.
Ramesh, C., Twatia, A. S. and Dhiya, M., 2002, Genetic variability and variability studies in garden pea. Haryana J. Hort. Sci., 31(3-4): 250-252.

Sharma, A., Sood, M., Rana, A. and Singh, Y., 2007, Genetic variability and association studies for green pod yield and component horticultural traits in garden pea under high hill dry temperate conditions. Indian J. of Hort., 64(4): 410-414.

Sharma, B. B. and Sharma, V. K., 2013, Character association and path analysis studies for yield and horticultural traits in garden pea. Envt. and Eco., 30(4A): 1591-1598.

Singh, A. K., 1995, Genetic variability and heritability study in pea (Pisum sativum L.). Crop Res., 10(2): 171-173.

Singh, A., Singh, S. and Babu, J. D. P., 2011, Heritability, character association and path analysis studies in early segregating population of field pea (Pisum sativum L.). Int. J. of Pl. Breed. and Genetics., 26 (5): 86-92.

Singh, A., Singh, S. and Babu, J. D. P., 2011, Heritability, character association and path analysis studies in early segregating population of field pea (Pisum sativum L.). Int. J. Breed. Genetics., 26 (5): 86-92.

Singh, G., Dev, S. K. and Singh, S. P., 2003, Genetic divergence in pea (Pisum sativum L.). Legume Res., 26(2): 131133.

Singh, R. K. and Choudhary, B. D., 1979, Book- Biometrical methods in quantitative genetic analysis. Page: 210214.

Singh, S., Vikas, P. and Singh, S. P., 1993, Variability and correlation studies in pea. Ann. Agri. Biol. Res., 4(1): 87-91.

Singh, S., Vikas, P. and Singh, S. P., 1993, Variability and correlation studies in pea. Ann. Agri. Biol. Res., 4(1): 87-91. 
Sureja, A. K., Singh, M. and Sharma, R. R., 2000, Genetic variability and heritability studies in garden pea (Pisum sativum L.). Indian J. Hort., 57(3): 243247.
Tiwari, G. and Roopalavanya, G., 2012, Genetic variability, character association and component analysis in $\mathrm{F}_{4}$ generation of field pea (Pisum sativum var. arvense L.). Karnataka J. Agric. Sci., 25 (2): 173-175.

\section{How to cite this article:}

Imran Ali, R.V. Patil, P.R. Dharmatti and Sridevi, O. 2018. Study on Genetic Parameters and Performance of Garden Pea (Pisum sativum L.) Genotypes for Yield and its Components (Under Northern Transitional Belt of Karnataka, India). Int.J.Curr.Microbiol.App.Sci. 7(09): 986-999. doi: https://doi.org/10.20546/ijcmas.2018.709.118 